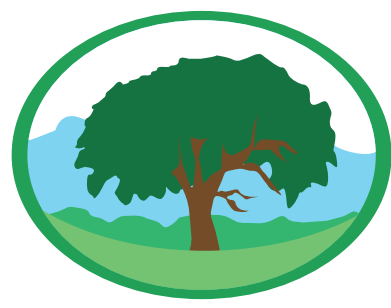

\title{
MODELAGEM HIDROLÓGICA DETERMINÍSTICA CHUVA-VAZÃO EM BACIAS HIDROGRÁFICAS: UMA ABORDAGEM INTRODUTÓRIA
}

\author{
CALDEIRA, T. L. ${ }^{\text {, }, ~ O L I V E I R A, ~ V . ~ A . ~}{ }^{2}$; STEINMETZ, A. A. ${ }^{2}$; VIOLA, M. R. ${ }^{3}$; BESKOW, S. ${ }^{4}$ \\ ${ }^{1}$ Universidade Federal de Pelotas/Centro de Engenharias. \\ ${ }^{2}$ Universidade Federal de Pelotas/Programa de Pós-Graduação em Recursos Hídricos. \\ ${ }^{3}$ Universidade Federal de Lavras/Departamento de Engenharia. \\ «Universidade Federal de Pelotas/Centro de Desenvolvimento Tecnológico.
}

Palavras-chave: modelos hidrológicos; análise de sensibilidade; calibração; validação.

\begin{abstract}
Resumo
A modelagem hidrológica em bacias hidrográficas consiste numa das principais e mais modernas ferramentas para gestão de recursos hídricos e dimensionamentos hidrológicos, no entanto, vários são os questionamentos quando estudantes e profissionais se deparam com aplicações práticas de um modelo hidrológico. Neste contexto, a presente Revisão de Literatura tem por objetivo apresentar, em língua portuguesa, uma breve visão introdutória às definições e aos conceitos básicos de modelagem hidrológica, a fim de fomentar sua compreensão e aplicações. São abordados neste artigo conceitos, classificações, objetivos da modelagem e as etapas envolvidas na utilização de modelos hidrológicos. É dado maior enfoque ao último tópico, justamente por ser na prática que o leitor deparar-se-á com terminologias específicas e a necessidade de optar por uma dentre várias metodologias existentes. Espera-se, com este texto, reduzir a distância entre a teoria e a prática, estimulando o leitor a lançar mão da técnica de modelagem hidrológica em tarefas profissionais diárias em universidades e empresas públicas ou privadas.
\end{abstract}

\section{DETERMINISTIC RAINFALL-RUNOFF HYDROLOGICAL MODELING IN WATERSHEDS: AN INTRODUCTORY LOOK}

Keywords: Hydrological models; sensitivity analysis; calibration; validation.

\begin{abstract}
Hydrological modeling in watersheds is one of the main and most modern tools applied to water resources management and hydrological engineering projects. However, several questions are raised when students and professionals need to deal with practical applications of a hydrological model. In this context, the objective of this literature review is to present in Portuguese a brief introductory look at some basic definitions and concepts associated with hydrological modeling in order to support its understanding and applications. Concepts, classifications, modeling objectives and steps involved in the use of hydrological models are discussed in this article. A greater focus is given to the latter topic because readers will commonly face specific terminologies and need to choose one among several existing methodologies. With this article, we hope to reduce the distance between theory and practice, thereby stimulating readers to use the hydrological modeling technique in their everyday professional tasks in universities and public or private companies.
\end{abstract}




\section{INTRODUC̣ÃO}

A água é um recurso natural indispensável à vida e estratégico como insumo ao desenvolvimento econômico de uma regiáo. No entanto, o demasiado crescimento populacional tem alarmado diferentes esferas da sociedade no que tange à sua oferta em quantidade e qualidade. Frente a este cenário, e considerando a disponibilidade hídrica variável no tempo e no espaço, o monitoramento hidrológico passa a ser fundamental, possibilitando o melhor gerenciamento dos recursos hídricos.

No Brasil, a rede hidrológica básica, cuja responsável é a Agência Nacional de Águas (ANA), geralmente contempla bacias hidrográficas de médio a grande porte, haja vista que nelas ocorrem os principais aproveitamentos hídricos, sobretudo hidrelétricos. Pequenas bacias hidrográficas notoriamente são pouco monitoradas e, comumente, os técnicos se deparam com ausência de dados no local de interesse.

Neste sentido, destaca-se a técnica de regionalização hidrológica, a qual permite transpor informaçóes de bacias hidrográficas monitoradas para o local de interesse, dentro de uma região hidrologicamente homogênea, não monitorado ou com série histórica de curto período de tempo (BESKOW et al., 2016a). Esta técnica atende demandas como estimativa de vazão média de longo termo, vazão máxima anual, vazóes de referência da curva de permanência, etc., no entanto, apresenta dificuldades e limitaçóes, principalmente associadas à necessidade de se ter uma região hidrologicamente homogênea e à inviabilidade de transposição da cronologia de ocorrência dos dados monitorados, isto é, de fornecer informações em uma escala temporal mais detalhada.

A ausência de monitoramento de vazão no local de interesse, bem como as limitaçôes intrínsecas à técnica de regionalização hidrológica e a necessidade de simulação de alteraçôes do sistema, têm impulsionado, nas últimas décadas, o desenvolvimento de modelos hidrológicos como subsídio à gestão de recursos hídricos.

Com o objetivo de descrever quantitativamente os componentes do ciclo hidrológico e a interação destes em uma bacia hidrográfica, os modelos estão sendo cada vez mais utilizados como ferramenta para gestão de recursos hídricos e tomada de decisão (BESKOW et al., 2016b), podendo ser empregados para inúmeras finalidades, tais como: geração de série sintética de vazões em cursos d'água não monitorados, preenchimento de falhas em séries históricas, previsão de inundação em tempo real, estimativa de frequência de vazôes de cheia, dimensionamentos hidrológicos de estruturas hidráulicas, análise de vazôes de referência para outorga de uso da água, avaliação do impacto das mudanças climáticas e de uso do solo sobre os recursos hídricos, estudos sobre serviços ecossistêmicos de caráter hidrológico, vazão ecológica, etc.

Neste contexto, o leitor geralmente fica curioso com algumas perguntas, como "Mas, afinal, o que é um modelo hidrológico?" ou "O que diferencia os diversos modelos existentes hoje?" ou ainda "Como faço para, de fato, utilizar um modelo hidrológico?". A presente revisão de literatura vem ao encontro a questionamentos como esses, com o objetivo de apresentar uma abordagem introdutória às definiçóes e aos conceitos básicos de modelagem hidrológica, a fim de fomentar a compreensão, utilização e aplicação de modelos hidrológicos no âmbito tanto científico quanto profissional.

\section{DEFINIC̣ÕES E CONCEITOS INICIAIS}

Em estudos de modelagem hidrológica são encontradas diversas terminologias específicas. Desta forma, inicialmente, torna-se necessário diferenciar sistema, modelo e simulação.

Um sistema, segundo definição de Dooge (1973), é uma estrutura, esquema ou procedimento, real ou abstrato, que num tempo de referência inter-relacionase com uma entrada, causa ou estímulo de energia ou informação, e uma saída, efeito ou resposta de energia ou informação.

Um modelo é a representação simplificada do comportamento de um sistema real natural ou artificial (SOROOSHIAN et al., 2008), e pode ser classificado como físico, analógico ou matemático. Modelos físicos, segundo Tucci (2005), representam o sistema por um protótipo em escala reduzida, enquanto que os modelos analógicos fazem uso de analogias de equaçóes que regem diversos fenômenos, tendo em vista modelar 
o processo desejado no sistema mais conveniente. Já os modelos matemáticos representam a natureza do sistema por meio de um conjunto de equaçóes ou sequências lógicas e, segundo Tucci (2005), são mais versáteis, pois permitem analisar diferentes situaçóes em um mesmo sistema ou em diferentes sistemas. Entretanto, podem apresentar desvantagens, haja vista as dificuldades intrínsecas à discretização de processos contínuos e à representação matemática de alguns processos físicos.

Em modelagem hidrológica, entende-se por fenômeno um processo físico que produz alteração de estado do sistema, por variável um valor que descreve quantitativamente um fenômeno e por parâmetro um valor que caracteriza o sistema, podendo variar no tempo e, ou, no espaço (TUCCI, 2005).

Considerando a bacia hidrográfica como um sistema parametrizado em função, basicamente, dos solos, vegetaçáo, clima e relevo, pode-se entender a simulação chuva-vazão como a aplicação de um modelo hidrológico matemático para a representação dos principais componentes do ciclo hidrológico desde a ocorrência da precipitação até a resposta da bacia em termos de vazões. Assim, é simulado o escoamento (fenômeno) pela quantificação da vazão (variável), dado um evento climático (entrada) com base na parametrização do sistema (bacia hidrográfica).

\section{CLASSIFICAC̣ÃO DE MODELOS HIDROLÓGICOS}

Os modelos hidrológicos podem ser classificados sob diferentes aspectos, todavia, comumente são empregadas classificaçóes de acordo com o tipo de variáveis utilizadas na modelagem (estocásticos ou determinísticos), a sua formulação (empíricos ou conceituais), a forma de representação dos dados (discretos ou contínuos), a consideração ou não da variabilidade espacial dos parâmetros na bacia hidrográfica (concentrados ou distribuídos) e a consideração de dependência temporal (estacionários ou dinâmicos) (MOREIRA, 2005).

Um modelo hidrológico é dito estocástico quando, segundo Shaw (1994), emprega distribuiçôes de probabilidade para gerar séries temporais de variáveis hidrológicas, tais como vazão, evaporaçáo, etc. Já o modelo determinístico é aquele que, segundo o autor, transforma a chuva em vazão através da quantificação dos processos físicos que ocorrem na bacia. Em outras palavras, Wheater (2008) conceitua modelos estocásticos como aqueles que, em decorrência da componente aleatória, podem produzir valores de saída diferentes para um mesmo conjunto de valores de entrada; modelos determinísticos, no entanto, devem, necessariamente, produzir os mesmos valores de saída para um mesmo conjunto de valores de entrada.

Os modelos hidrológicos também podem ser classificados quanto à formulação em conceituais ou empíricos, segundo Tucci (2005). Tal autor relata que os modelos são ditos conceituais quando as funçôes empregadas para representar o comportamento do sistema levam em consideraçáo os processos físicos, quando não consideram estes processos, o modelos são ditos empíricos, ou "caixas-preta”. No entanto, uma vez que as funçóes empíricas associadas aos componentes físicos são frequentemente usadas em modelos conceituais, alguns hidrólogos preferem classificar os modelos como semi-conceituais ou fisicamente baseados. $\mathrm{O}$ primeiro inclui as características dos processsos, mas os parâmetros das equaçóes tem um nível razoável de empirismo; o segundo emprega equaçóes diferenciais para representar os processos, sendo seus parâmetros mais representativos da realidade física (BESKOW, 2009).

Em relação à escala temporal, os modelos hidrológicos são classificados como "modelos baseados em eventos", também denominados de "modelos discretos", ou como "modelos contínuos" (WHEATER, 2008). Os modelos baseados em eventos objetivam a modelagem de períodos isolados de uma série histórica, buscando, por exemplo, representar eventos de cheia ou de recessão; já os modelos contínuos objetivam a modelagem de longos períodos, geralmente contemplando diferentes comportamentos hidrológicos ocorrentes ao longo do ano hidrológico (MARINHO FILHO et al., 2012).

A discretização espacial, por sua vez, está relacionada à área na qual as variáveis são consideradas homogêneas para finalidade de modelagem, podendo classificar o modelo como concentrado ou distribuído. Nos 
modelos hidrológicos com abordagem concentrada, os parâmetros, as entradas e as saídas são valores médios únicos que visam representar a bacia em sua totalidade (WHEATER, 2008). Tal abordagem limita a representação da variabilidade espacial das características da bacia, haja vista a heterogeneidade natural dos solos, vegetação e relevo (HARTMANN; BALES; SOROOSHIAN, 1999). Nos modelos com abordagem distribuída, nos quais a bacia hidrográfica é representada por um grid de células, esta limitação é minimizada, pois levam em consideração a variabilidade espacial dos parâmetros, entradas e saídas; quando a bacia é dividida em sub-bacias, o modelo é dito semidistribuído. (WHEATER, 2008).

Os modelos hidrológicos podem ainda ser estacionários, descrevendo o fenômeno, em determinado momento, sem variação temporal dos parâmetros, ou dinâmicos, para os quais os parâmetros variam em função do tempo (MARINHO FILHO et al., 2012).

\section{ETAPAS DA MODELAGEM HIDROLÓGICA}

$\mathrm{Na}$ Ciência Hidrológica um modelo hidrológico é entendido como uma ferramenta que visa atingir a um determinado objetivo (TUCCI, 2005). De acordo com a Organizaçáo Mundial de Meteorologia (WMO, 2008), a escolha do modelo hidrológico deve considerar: (i) $\mathrm{O}$ objetivo geral da modelagem (e.g. avaliação de impactos de mudanças climáticas, previsão do regime hidrológico, entre outros); (ii) a variável hidrológica a ser modelada (ex: vazóes diárias, mensais, semanais, parâmetros de qualidade da água); (iii) as características climáticas e fisiográficas do sistema a ser modelado; (iv) a disponibilidade e qualidade de dados requeridos para calibração e operação do modelo; (v) a simplicidade do modelo, no que se refere à complexidade dos fenômenos hidrológicos e facilidade de aplicação; entre outros.

Após a definição do modelo, desenvolvem-se as etapas de análise de sensibilidade, calibração, validação e predição. Segundo White e Chaubey (2005) e Ma et al. (2012), a análise de sensibilidade é utilizada para identificar os parâmetros mais importantes do modelo aplicado à área de interesse e visa avaliar a influência da alteração do valor de diferentes parâmetros na resposta de uma variável de saída, sendo os parâmetros mais sensíveis, ou aqueles que exercem maior influência, empregados na etapa de calibraçáo.

A compreensão sobre todos os parâmetros de entrada de um modelo hidrológico, bem como dos processos que por ele são representados, é de suma importância para que a calibração dos parâmetros seja coerente (LENHART et al., 2002), sendo a acurácia dessas prediçôes altamente dependente do quão bem definidas são as estruturas do modelo e de como esses parâmetros são determinados (DUAN; SOROOSHIAN; GUPTA, 1994).

Desta forma, Castro (2013) relata que a delimitação de intervalos para variação dos parâmetros é extremamente importante para definir a sensibilidade; os limites de variaçáo devem ser definidos de acordo com as características da bacia hidrográfica analisada, caso contrário, parâmetros que não são sensíveis podem apresentar alta sensibilidade e parâmetros que são realmente importantes podem ser desconsiderados por apresentarem baixa sensibilidade.

Diversos métodos de análise de sensibilidade têm sido utilizados na modelagem hidrológica, podendo serem classificados como análise de sensibilidade local ou global, dos quais destaca-se o método de "exploração do espaço amostral do parâmetro". A análise de sensibilidade local é realizada variando-se um único parâmetro por vez ao redor de um pequeno espaço de um dado ponto amostral. Por outro lado, a análise de sensibilidade global considera a influência global de todos os parâmetros analisados na resposta da variável estimada pelo modelo, variando-os de acordo com seus intervalos de variaçáo previamente determinados (YUAN et al., 2015). De acordo com os autores, os métodos locais requerem um menor esforço computacional. Entretanto, seus resultados geralmente apresentam um maior nível de incerteza devido aos métodos locais serem aplicados somente ao redor do ponto amostrado, não sendo capazes de explicar a nãolinearidade, a não-monotonicidade e a interação entre os parâmetros do modelo. De acordo com Saltelli et al. (2008) os métodos globais geralmente minimizam as deficiências dos métodos locais.

Dentre os métodos de análise de sensibilidade local 
destaca-se o "One-at-a-time", que consiste na variação do valor de um parâmetro por vez enquanto todos os outros são mantidos fixos. Dentre os globais, destaca-se o método de Efeitos Elementares (Elementary Effects), no qual considera a variação de todos os parâmetros do espaço amostral e que possibilita, por meio de testes estatísticos, classificar os parâmetros que promovem maior influência na resposta da variável predita pelo modelo (YUAN etal., 2015). Uma aplicação do método "One-at-a-time" pode ser observada em Beskow Mello e Norton (2011), enquanto que do método dos Efeitos Elementares, em Gan et al. (2014).

A calibraçáo, por sua vez, consiste no ajuste, para a bacia hidrográfica em análise, de um conjunto de parâmetros cujos valores são desconhecidos ou indiretamente mensuráveis, indicados pela análise de sensibilidade. Consiste em um esforço para refinar os valores dos parâmetros de um modelo para um determinado conjunto de condiçôes locais, reduzindo, assim, a incerteza da etapa de predição. A calibração do modelo é feita selecionando cuidadosamente os valores para os parâmetros de entrada do modelo (dentro de suas respectivas faixas de incerteza), comparando previsóes do modelo (saída) com os dados observados para um determinado conjunto de condiçóes assumidas (ARNOLD et al., 2012).

De acordo com Her e Chaubey (2015), quando uma única variável de saída do modelo, como por exemplo a vazão, é associada à múltiplos processos e a muitos parâmetros, o número de parâmetros a serem calibrados tende a ser maior que o número de variáveis a serem comparadas na calibração, caracterizando a over-parameterization. Ao tentar ajustar um grande número de parâmetros a partir de um número limitado de observaçôes, segundo tais autores, a solução para a calibração tende a não ser única.

Neste sentido, destaca-se o conceito de equifinalidade, que é a existência de vários conjuntos de valores de parâmetros que proporcionam resultados satisfatórios e bastante semelhantes entre si, sendo impossível distinguir a melhor combinação (BEVEN, 2004). A equifinalidade, segundo Her e Chaubey (2015), vem sendo, nas últimas três décadas, bastante discutida na literatura associada à modelagem hidrológica (SOROOSHIAN; GUPTA,
1983; BEVEN; BINLEY, 1992; GAN; BIFTU, 1996; BEVEN; FREER, 2001; BEVEN, 2006; VRUGT; BEVEN, 2018).

Collischonn e Tucci (2003) corroboram ao destacar que, assim como podem ser obtidas várias combinações de parâmetros com justificativa técnica e resposta adequada para o problema, e entre as quais não é possível, em geral, distinguir a mais aceitável ou a melhor, podem existir soluçóes que atendam às equaçóes, mas não representam a realidade lógica do problema ou mesmo dos valores esperados dos parâmetros.

Her e Chaubey (2015) relatam que a equifinalidade pode ser mais severa no caso da calibração de modelos distribuídos, como o Soil and Water Assessment Toll (SWAT), o qual apresenta over-parameterization quando observações adicionais não são disponibilizadas. Frente ao exposto, ressalta-se o princípio da parcimônia, que é a representação adequada do comportamento de um processo ou de um sistema por um modelo com o menor número possível de parâmetros (TUCCI, 2005). Muitos estudos têm empregado este princípio para propor modelos hidrológicos com abordagem simplificada o suficiente para evitar over-parameterization e reduzir as incertezas (HER; CHAUBEY, 2015), como é o caso, por exemplo, do Lavras Simulation of Hydrology (LASH) (BESKOW et al., 2011; CALDEIRA, 2016) e do Topography Based Hydrological Model (TOPMODEL) (BEVEN; KIRKBY, 1979).

A etapa de calibração de parâmetros, segundo Zeckoski et al. (2015) e Daggupati et al. (2015), pode ser realizada, basicamente, por tentativa e erro (calibração manual) ou por métodos automáticos. Em ambos os casos, o objetivo é encontrar valores para um conjunto de variáveis de modo a representar os fenômenos naturais da forma mais realística, contudo, na primeira metodologia, são testadas diferentes combinaçóes, enquanto que na segunda, empregam-se métodos matemáticos e computacionais de otimização.

Segundo Jarvis e Larsbo (2012), a escolha do método de calibração depende, entre outros fatores, do objetivo do estudo, da natureza e qualidade dos dados de entrada e da capacidade computacional disponível.

No método por tentativa e erro, considerado pioneiro na calibração de modelos hidrológicos 
chuva-vazão devido à sua simplicidade, apesar de demorado e subjetivo, a eficácia é al tamente dependente da experiência do usuário (COLLISCHONN;TUCCI, 2003). Além disso, esse método não permite ao usuário obter informaçôes sobre a incerteza de parâmetros ou sua predição (JARVIS; LARSBO, 2012). O ajuste dos parâmetros, seguindo este método, ocorre de forma manual, em repetidas execuçóes do modelo e análises de resultados. A cada tentativa o usuário verifica, com base nos hidrogramas observado e calculado, o formato geral e o ajuste das vazóes mínimas e de cheias, podendo ainda observar os valores de algumas funçóes objetivo (COLLISCHONN; TUCCI, 2003).

Para minimizar as dificuldades de calibração intrínsecas ao método da tentativa e erro, técnicas automáticas de otimização de parâmetros têm sido propostas. Tais técnicas buscam os valores dos parâmetros de forma automática, proporcionando o melhor resultado para uma função dependente dessas variáveis dentro de um objetivo e obedecidas suas restrições (TUCCI, 2005). O procedimento comumente adotado na calibração automática de parâmetros emprega algoritmos de calibração, funçóes objetivo e critérios de parada.

Existem vários algoritmos de calibração disponíveis na literatura, como os de busca local, os de gradiente, os baseados na segunda derivada, os de otimização global, entre outros, destaca Šimůnek et al. (2012). No entanto, devido às dificuldades encontradas para o propósito de modelagem hidrológica empregando os métodos do gradiente e da segunda derivada, estes não têm sido utilizados em modelos conceituais.

Já os algoritmos de busca local eram os mais empregados, contudo, relatam Duan, Sorooshian e Gupta (1992), não foram desenvolvidos para lidar com alguns problemas associados à calibração de modelos conceituais. Segundo Collischonn e Tucci (2003), em duas aplicaçôes idênticas quanto à bacia hidrográfica e ao tempo de calibraçáo, estes algoritmos tendem a produzir valores diferentes para um mesmo conjunto de parâmetros, dependendo do ponto de partida da busca. Isso ocorre em virtude das superfícies de resposta das funçóes objetivo comumente utilizadas, as quais não são lineares e contínuas (DUAN; SOROOSHIAN; GUPTA, 1992; XIONG; O’CONNOR, 2000). Sobre essas superfícies de resposta, enfatizam Collischonn e Tucci (2003), as técnicas de calibração baseadas em busca local tendem a gerar resultados pouco confiáveis.

Com relação aos algoritmos de otimização global, Zeckoski et al. (2015) relatam que estes vêm sendo amplamente empregados na modelagem hidrológica, dentre os quais destacam-seo Shuffled Complex Evolution (SCE-UA) (DUAN; SOROOSHIAN; GUPTA, 1992), o Sequential Uncertainty Fitting - (SUFI) (ABBASPOUR; JOHNSON; VAN GENUCHTEN, 2004), o Generalized Likelihood Uncertainty Estimation (GLUE) (BEVEN; BINLEY, 1992) e o Parameter Solution (ParaSol) (VAN GRIENSVEN; BAUWENS, 2003).

Contudo, Daggupati et al. (2015) e Arnold et al. (2012) ressaltam que, apesar da calibração automática ser mais eficiente, quando comparada à calibração manual, o processo de otimizaçáo pode resultar em parâmetros calibrados que não refletem a realidade as características da área de estudo. Sendo assim, o conhecimento do usuário sobre a área de interesse é essencial na determinação de estimativas inicias dos intervalos de variação dos parâmetros.

$\mathrm{Na}$ aplicação de algortimos de calibração, uma das partes fundamentais é o estabelecimento da função objetivo, que é a representação matemática da qualidade dos resultados obtidos para um conjunto de parâmetros calibrados (COLLISCHONN; TUCCI, 2003). O algoritmo de calibração atribui valores ao conjunto de parâmetros e calcula a função objetivo, seguindo em busca do valor ótimo até que seja constatada a convergência dos valores dos parâmetros, quando o processo iterativo encerra-se. $\mathrm{O}$ critério de parada do processo iterativo da calibração automática pode ser definido, segundo Sorooshian e Gupta (1995), pela convergência da funçáo objetivo, pela convergência dos valores dos parâmetros ou por um número máximo de iterações.

O estabelecimento da função objetivo é totalmente dependente da finalidade a que se propóe a modelagem hidrológica. A modelagem hidrológica objetiva aproximar o máximo possível o hidrograma calculado a partir do modelo àquele observado na seção de controle, devendo a função objetivo, neste caso, medir a discrepância entre os valores para que possa 
ser minimizada (TUCCI, 2005). A função quadrática da diferença das vazóes geralmente apresenta melhores resultados para esta aplicação, ressaltam Collischonn e Tucci (2003), contudo, pode gerar resultados tendenciosos, pois tende a priorizar os valores maiores a medida que se minimiza o desvio quadrático das vazões.

Quando é otimizado o valor de uma única função objetivo, o processo de calibração automática é denominado monobjetivo, entretanto, quando otimizado o valor de duas ou mais funçôes-objetivo simultaneamente, o processo de calibração automática é denominado multiobjetivo (COLLISCHON; TUCCI, 2003)

Outra importante consideração sobre a etapa de calibração de modelos hidrológicos concerne aos dados empregados. Segundo Perrin et al. (2007), existem poucas recomendaçôes sobre o quão longa uma série de dados de vazão deve ser para gerar resultados satisfatórios na calibração, sendo comumente considerado que "quanto maior, melhor", ou então, de dois a dez anos de observaçóes.

Sorooshian, Gupta e Fulton (1983) relatam que o conteúdo da informação é mais importante que a quantidade de dados, pois até mesmo métodos robustos de calibração não conseguiriam extrair informaçóes que não estão presentes. Os autores sugerem que seja empregado, no mínimo, um ano hidrológico completo de dados observados para calibraçáo de modelos chuvavazão.

Por sua vez, Perrin et al. (2007), calibrando modelos GR4J ( PERRIN; MICHEL; ANDRÉASSIAN, 2003) e TOPMO (MICHEL; PERRIN; ANDRÉASSIAN, 2003), versão modificada do TOPMODEL (BEVEN; KIRKBY, 1979), para doze bacias hidrográficas americanas, com várias características hidrológicas e climáticas, constataram que, de modo geral, 350 dias, escolhidos aleatoriamente em um longo período de tempo, são suficientes para obter uma estimativa robusta de parâmetros.

Via de regra, a definição do período de dados a ser empregado na calibração de parâmetros de um modelo hidrológico está atrelada à disponibilidade e qualidade de dados observados, sendo bastante variável na literatura.
As variáveis temporais de entrada, comumente empregadas em modelos hidrológicos chuva-vazão, são a precipitação, a evapotranspiração e a vazão, sendo esta empregada no processo de calibração do modelo hidrológico (TUCCI, 2005). De acordo com Maidment (1993), tais variáveis, por derivarem de monitoramento, geralmente são observadas em intervalos de tempo pré-definidos, não sendo conhecidas suas variaçóes entre observaçóes, o que implica na aceitação de uma variação média no intervalo.

Segundo Viola (2011), a discretização temporal, também denominada passo do modelo, está diretamente relacionada à acurácia desejada e à disponibilidade de dados. A rede hidrométrica nacional gerenciada pela ANA, por exemplo, disponibiliza dados de vazáo média diária, o que é um fator limitante para calibração de modelos em bacias hidrográficas com tempo de concentração inferior à um dia.

O processo de validação de um modelo hidrológico, por sua vez, envolve a simulação do modelo utilizando os valores de parâmetros determinados durante o processo de calibração a fim de conferir a adequabilidade do mesmo aplicado à área de estudo. Segundo Zeckoski et al. (2015), em geral, métodos de comparação gráfica e métodos estatísticos são utilizados para aferir a acurácia da simulação. Klemeš (1986) apresenta quatro metodologias para validação de modelos hidrológicos: Split sample test, Proxy basin, Differential Split-sample e Proxy-basin differential split-sample. Tais metodologias são descritas a seguir, em ordem crescente de complexidade.

O teste Split sample é empregado na validação de modelos para aplicaçáo a processos estacionários com calibração e simulação na mesma bacia, como é o caso do preenchimento de períodos falhos em série histórica fluviométrica. Este teste consiste na aplicação de dois períodos de tempo distintos: um período para calibrar o modelo hidrológico e outro para aplicar os parâmetros obtidos com a calibração. O modelo deve apresentar resultados satisfatórios em ambos os períodos. Cabe ressaltar que uma série histórica estacionária, de acordo com Tucci (2005), é caracterizada pela continuidade dos valores dos parâmetros estatísticos ao longo do tempo.

O teste Proxy basiné utilizado para validar a aplicação 
do modelo a processos estacionários com calibração e simulação em bacias distintas, como, por exemplo, na geração de séries históricas em bacias hidrográficas sem monitoramento fluviométrico. Nessa situação, é recomendado que sejam empregadas duas bacias hidrográficas A e B com características edafoclimáticas semelhantes à da bacia $\mathrm{C}$ onde deseja-se obter os dados, utilizando uma para calibração e outra para validação dos parâmetros calibrados, devendo-se observar bons resultados.

O teste Differential Split-sample é empregado na validaçáo do modelo para aplicaçáo a processos não estacionários, com calibração e simulação na mesma bacia. A aplicação deste teste se dá em bacias hidrográficas monitoradas, para validar modelos para a simulação de mudanças no regime fluviométrico em decorrência de mudanças climáticas ou de uso do solo.

Se o objetivo for verificar o impacto das alteraçóes do regime pluvial, por exemplo, devem ser identificados, nos registros históricos existentes, um período chuvoso e outro seco, caracterizados por valores acima e abaixo da média, respectivamente. $\mathrm{O}$ procedimento de calibração e aplicação do modelo calibrado, para o estabelecimento das estatísticas que permitem avaliálo, depende das aplicaçóes pretendidas. Se o objetivo for avaliar os impactos de uma redução do regime pluvial, o modelo deve ser calibrado com dados do período chuvoso e aplicado no período seco, ou viceversa, obtendo-se boas estatísticas.

Já a validação para a simulação de alteraçôes do regime fluvial devido a mudanças no uso do solo requer o conhecimento histórico de uso do solo na bacia, buscando identificar um período prévio à mudança no uso do solo e outro posterior. O teste consiste na calibração do modelo no período prévio às alteraçôes, e aplicação, com as devidas modificaçóes nos parâmetros atrelados à cobertura vegetal, no período após as alterações, esperando-se bons resultados.

O Proxy-basin differential split-sample teste é empregado na validação de modelos para aplicação em processos não estacionários calibrados e simulados em bacias distintas, como no caso da validação de modelos hidrológicos para aplicação em simulação de mudanças hidrológicas em bacias hidrográficas desprovidas de monitoramento hidrológico. De modo semelhante ao
Proxy basin, é necessário empregar dados de duas bacias hidrográficas A e B com características edafoclimáticas semelhantes à da bacia $\mathrm{C}$ sem registros históricos.

No caso da validação para utilização em alteraçóes de regime pluvial, é necessário identificar períodos chuvosos e secos na bacia $A\left(A_{\text {chuvoso }}\right.$ e $\left.A_{\text {seco }}\right)$ e na bacia $B$ $\left(\mathrm{B}_{\text {chuvoso }}\right.$ e $\left.\mathrm{B}_{\text {seco }}\right)$. Se o objetivo for validar o modelo para aplicação a um cenário de baixas precipitaçóes, deve-se realizar a calibração com o período $\mathrm{A}_{\text {chuvoso }}$ e aplicação com o $\mathrm{B}_{\text {seco }}$ e, posteriormente, calibração com $\mathrm{B}_{\text {chuvoso }} \mathrm{e}$ aplicaçáo com $\mathrm{A}_{\text {seco }}$, e encontrar bons resultados.

Segundo Daggupati et al. (2015), a validação de modelos hidrológicos chuva-vazáo tem sido realizada com o emprego do teste Split sample, contudo, alguns trabalhos têm aplicado também o Proxy basin. No entanto, a definição do teste a ser empregado na validação é função principalmente do objetivo da análise, como no estudo de Magand et al. (2015), os quais empregaram o teste Differential Split-sample para validar o Catchment Land Surface Model (CLSM) para a bacia hidrográfica do alto Durance, localizada nos Alpes Franceses, objetivando a simulação de cenário de mudanças climáticas.

Os principais coeficientes estatísticos utilizados nos testes para avaliação do desempenho dos modelos na calibração e validação são: o coeficiente de determinação $\left(\mathrm{R}^{2}\right)$, o viés percentual de vazóes (PBIAS) (Gupta et al., 1999), a raíz quadrada do erro quadrático médio (RMSE) e o coeficiente de Nash e Sutcliffe $\left(\mathrm{C}_{\mathrm{NS}}\right)$ (NASH; SUTCLIFFE, 1970). Para modelagem hidrológica ao passo mensal, por exemplo, Moriasi et al. (2007) sugerem a seguinte classificação para $\mathrm{C}_{\mathrm{NS}}$ : $\mathrm{C}_{\mathrm{NS}}>0,75$, muito bom; $0,65<\mathrm{C}_{\mathrm{NS}}<0,75$, bom; $0,50<$ $\mathrm{C}_{\mathrm{NS}}<0,65$, satisfatório; $\mathrm{C}_{\mathrm{NS}}<0,50$, insatisfatório.

Uma vez constatado, na etapa de validação, que o modelo consegue representar o comportamento hidrológico da bacia hidrográfica, o mesmo está apto a ser empregado para predição. Nesta etapa, buscar-se-á atingir o objetivo principal da modelagem hidrológica: utilizar o modelo - calibrado e validado - como ferramenta de suporte à tomada de decisão, tendo em vista os inúmeros exemplos de aplicação mencionados na seção anterior. 


\section{CONSIDERAC̣̃̃ES FINAIS}

A presente Revisão de Literatura buscou oferecer ao leitor uma abordagem introdutória - e em língua portuguesa - sobre os principais questionamentos acerca da modelagem hidrológica determinística chuva-vazão em bacias hidrográficas. Para tal intento, foram apresentados alguns conceitos necessários ao entendimento da modelagem hidrológica, as classificações mais empregadas quando se refere a modelos hidrológicos, e as principais etapas envolvidas na utilizaçáo de um modelo para representar o comportamento de uma bacia hidrográfica. Este último tópico foi mais explorado pelos autores, justamente porque é na utilização de um modelo hidrológico que o leitor deparar-se-á com terminologias específicas e a necessidade de optar por uma metodologia ou outra. Espera-se com este texto reduzir a distância entre a teoria e a prática, estimulando o leitor a lançar mão da técnica de modelagem hidrológica em seu cotidiano profissional, quer seja na academia, empresas públicas ou privadas.

\section{REFERÊNCIAS}

ARNOLD, J. G.; MORIASI, D. N.; GASSMAN, P. W.; ABBASPOUR, K. C.; WHITE, M. J.; SRINIVASAN, R.; SANTHI, C.; HARMEL, R. D.; VAN GRIENSVEN, A.; VAN LIEW, M. W.; KANNAN, N.; JHA, M. K. SWAT: Model use, calibration, and validation. Transactions of the ASABE, v. 55, n. 4, p. 1494-1508, 2012.

ABBASPOUR, K. C.; JOHNSON, C. A.; VAN GENUCHTEN, M. T. Estimating uncertain flow and transport parameters using a sequential uncertainty fitting procedure. Vadose Zone Journal, Madison, v. 3, n. 4. p. 1340-1352, 2004.

BESKOW, S. LASH Model: a hydrological simulation tool in GIS framework. Tese (Doutorado em Engenharia Agrícola). Universidade Federal de Lavras. Lavras, p. 118. 2009.

BESKOW, S.; MELLO, C. R. D.; NORTON, L. D. Development, sensitivity and uncertainty analysis of LASH model. Scientia Agricola, v. 68, p. 265-274, 2011a." para "BESKOW, S.; MELLO, C. R. D.; NORTON, L. D. Development, sensitivity and uncertainty analysis of LASH model. Scientia Agricola, v.68, p. 265-274, 2011.

BESKOW, S.; MELLO, C. R.; NORTON, L. D.; SILVA, A. M. Performance of a distributed semi-conceptual hydrological model under tropical watershed conditions. Catena, v. 86, n. 3, p. 160$171,2011$.

BESKOW, S.; MELLO, C. R.; VARGAS, M. M.; CALDEIRA, T. L.; DURÃES, M. F.; AGUIAR, M. S. Artificial intelligence techniques coupled with seasonality measures for hydrological regionalization of Q90 under Brazilian conditions. Journal of Hydrology, v. 541, p. 1406-1419, 2016 a.

BESKOW, S.; TIMM, L. C.; TAVARES, V. E. Q.; CALDEIRA,
T. L.; AQUINO, L. S. Potential of the LASH model for water resources management in data-scarce basins: a case study of the Fragata River basin, Southern Brazil. Hydrological Sciences Journal, v. 61, p. 2567-2578, 2016 b.

BEVEN, K. J. Rainfall-runoff modelling: the primer. 1'a. ed. Chichester: John Wiley \& Sons, 2004.

BEVEN, K. J. A manifesto for equifinality thesis. Journal of Hydrology, v. 320, n. 1-2, p. 18-36, 2006.

BEVEN, K. J.; BINLEY, A. The future of distributed models: Model calibration and uncertainty prediction. Hydrological Processes, v. 6, p. 279-298, 1992.

BEVEN, K. J.; FREER, J. Equifinality, data assimilation, and uncertainty estimation in mechanistic modeling of complex environmental systems using the GLUE methodology. Journal of Hydrology, v. 249, n. 1-4, p. 11-29, 2001.

BEVEN, K. J.; KIRKBY, M. J. A physically-based, variable contributing area model of basin hydrology. Hydrological Sciences Bulletin, v. 24, n. 1, p. 43-69, 1979.

CALDEIRA, T. L. Aprimoramento computacional do modelo Lavras Simulation of Hydrology (LASH): aplicação em duas bacias do Rio Grande do Sul. Pelotas: Dissertação (Mestrado em Recursos Hídricos). Universidade Federal de Pelotas. Pelotas, p. 213. 2016.

CASTRO, C. B. Avaliação do modelo SWAT na simulação da vazão em bacia agrícola do Cerrado intensamente monitorada. Dissertação (Mestrado em Geoprocessamento e Análise Ambiental). Universidade de Brasília. Brasília, p. 122. 2013.

COLlisCHONN, W. Simulação Hidrológica de Grandes Bacias. Tese (Doutorado em Engenharia). Universidade Federal do Rio Grande do Sul. Porto Alegre, p. 194. 2001.

COLLISCHONN, W.; TUCCI, C. E. M. Ajuste multiobjetivo dos parâmetros de um modelo hidrológico. Revista Brasileira de Recursos Hídricos, v. 8, n. 3, p. 27-41, 2003.

DAGGUPATI, P.; PAI, N.; ALE, S.; DOUGLAS-MANKIN, K. R.; ZECKOSKI, R. W.; JEONG, J. PARAJULI, P. B.; SARASWAT, D.; YOUSSEF, M. A. A recommended calibration and validation strateggy for hydrologic and water quality models. Transactions of the ASABE, v. 58, n. 6, p. 1705-1719, 2015.

DOOGE, J. Linear Theory of Hydrological Systems. Technical Bulletin n. 1468. Washington DC: Agricultural Research Service - US Department of Agriculture, 1973. 327 p.

DUAN, Q.; SOROOSHIAN, S.; GUPTA, V. Effective and Efficient Global Optimization for Conceptual Rainfall-Runoff Models. Water Resources Research, v. 28, n. 4, p. 1015-1031, 1992.

GAN, T. Y.; BIFTU, G. F. Automatic calibration of conceptual rainfall-runoff models: Optimization algorithms, catchment conditions, and model structure. Water Resources Research, v. 32, n. 12, p. 3513-3524, 1996.

GAN, Y.; DUAN, Q.; GONG, W.; TONG, C.; SUN, Y.; CHU, W.; YE, A.; MIAO, C.; ZHENHUA, D. A comprehensive evaluation of various sensitivity analysis methods: A case study with a hydrological model. Environmental Modelling \& Software, v. 51, p. 269-285, 2014.

GUPTA, H. V., SOROOSHIAN, S., \& YAPO, P. O. Status of automatic calibration for hydrologic models: Comparison with multilevel expert calibration. Journal of. Hydrological Engineering, v, 4, n. 2, p. 135-143, 1999.

HARTMANN, H. C.; BALES, R.; SOROOSHIAN, S. Weather, climate and hydrologic forecasting for the southwest U.S. Tucson: The University of Arizona, 1999. 172 p. 
HER, Y.; CHAUBEY, I. Impact of the numbers of observations and calibration parameters on equifinality, model performance, and output and parameter uncertainty. Hydrological Processes, v. 29, n. 19, p. 4220-4237, 2015.

JARVIS, N.; LARSBO. M. MACRO (v5.2): Model use, calibration, and validation. Trans. ASABE, v. 55, n. 4, p. 14131423, 2012.

KLEMEŠ, V. Operational testing of hydrological simulation. Hydrological Sciences Journal, v. 31, n. 1, p. 13-24, 1986.

LENHART, T.; ECKHARDT, K.; FOHRER, N.; FREDE, H. G. Comparison of two different approaches of sensitivity analysis. Physics and Chemistry of the Earth, v. 27, n. 9-10, p. 645-654, 2002.

MA, L., AHUJA, L. R., NOLAN, B. T., MALONE, R. W., TROUT, T. J., QI, Z. Root Zone Water Quality Model (RZWQM2): Model use, calibration, and validation. Transactions of the ASABE, v. 55, n. 4, p. 1425-1446, 2012.

MAGAND, C.; DUCHARNE, A.; LE MOINE, N.; BRIGODE, P. Parameter transferability under changing climate: case study with a land surface model in the Durance watershed, France. Hydrological Sciences Journal, v. 60, n. 7-8, p. 1408-1423, 2015. MAIDMENT, D. R. GIS and hydrologic modeling. In: GOODCHILD, M. F.; PARKS, B. O.; STEYAERT, L. T. Environmental modeling with GIS. New York: Oxford University, 1993. Cap. 14, p. 147-167.

MARINHO FILHO, G. M.; ANDRADE, R. S.; ZUKOWSKI, J. C.; MAGALHÁES, L. L. Modelos hidrológicos: conceitos e aplicabilidades. Revista de Ciências Ambientais, v. 6, n. 2, p. $35-$ 47, 2012.

MELLO, C. R.; NORTON, L. D.; PINTO, L. C.; BESKOW, S.; CURI, N. Agricultural watershed modeling: a review for hydrology and soil erosion processes. Ciência \& Agrotecnologia, v. 40, n. 1, 2016.

MICHEL, C.; PERRIN, C.; ANDRÉASSIAN, V. The exponential store: a correct formulation for rainfall-runoff modelling. Hydrological Sciences Journal, v. 48, n. 1, p. 109124, 2003.

MOREIRA, I. A. Modelagem hidrológica chuva-vazão com dados de radar e pluviômetros. Dissertação (Mestrado em Engenharia Hidrológica). Universidade Federal do Paraná. Curitiba, p. 81. 2005.

MORIASI, D. N.; ARNOLD, J. G.; VAN LIEW, M. W.; BINGNER, R. L.; HARMEL, R. D.; VEITH, T. L. Model evaluation guidelines for systematic quantification of accuracy in watershed simulation. Transactions of the ASABE, v. 50, p. 885900, 2007.

NASH, J. E.; SUTCLIFFE, J. V. River flow forecasting through conceptual models I: a discussion of principles. Journal of Hydrology, v. 10, n. 3, p. 282-298, 1970.

PERRIN, C.; OUDIN, L.; ANDREASSIAN, V.; ROJASSERNA, C.; MICHEL, C.; MATHEVET, T. Impact of limited streamflow data on the efficiency and the parameters of rainfallrunoff models. Hydrological Sciences Journal, v. 52, n. 1, p. 131 151, 2007.

PERRIN, C.; MICHEL, C.; ANDRÉASSIAN, V. Improvement of a parsimonious model for streamflow simulation. Journal of Hydrology, v. 279, n. 1/4, p. 275-289, 2003.

SALTELLI, A., RATTO, M., ANDRES, T., CAMPOLONGO, F., CARIBONI, J., GATELLI, D., \& TARANTOLA, S. Global Sensitivity Analysis: The Primer. Hoboken, N.J.: John Wiley and Sons, 2008. 304 p.
ŠIMU゚NEK, J.; VAN GENUCHTEN; M. T.; ŠEJNA, M. HYDRUS: Model use, calibration, and validation. Trans. ASABE, v. 55, n. 4: 1261-1274, 2012.

SOROOSHIAN, S.; GUPTA, V. K. Automatic calibration of conceptual rainfall-runoff models: The question of parameter observability and uniqueness. Water Resources Research, v. 19, n. 1 , p. 260-268, 1983.

SOROOSHIAN, S.; GUPTA, V. K. Model calibration. In: SINGH, V. J. Computer models of watershed hydrology. Highlands Ranch: Water Resources Publications, 1995.

SOROOSHIAN, S.; GUPTA, V. K.; FULTON, J. Evaluation of Maximum Likelihood Parameter Estimation Techniques for Conceptual Rainfall-Runoff Models: Influence of Calibration Data Variability and Length on Model Credibility. Water Resources Reserach, v. 19, n. 1, p. 251-259, 1983.

SOROOSHIAN, S.; HSU, K.; COPPOLA, E.; TOMASSETTI, B. VERDECCHIA, M. VISCONTI, G. Hydrological modelling and the water cycle. Springer: Heidelberg, 2008. 291 p.

VRUGT; J. A.; BEVEN, K. J. Embracing equifinality with efficiency: Limits of Acceptability sampling using the DREAM(LOA) algorithm. Journal of Hydrology, v. 559, p. 954971, 2018.

TUCCI, C. E. M. Modelos Hidrológicos. 2a . ed. Porto Alegre: UFRGS/ABRH, 2005. 678 p.

VAN GRIENSVEN, A.; MEIXNER, T.; GRUNWALD, S.; BISHOP, T.; DILUZIO, M.; SRINIVASAN, R. A global sensitivity analysis tool for the parameters of multi-variable catchment models. Journal of Hydrology, v. 324, n. 1-4, p. 1023, 2006.

VAN GRIENSVEN, A.; BAUWENS, W. Multi-objective autocalibration for semi-distributed water quality models. Water Resources Research, Washington, v. 39, n. 12, p. 1348, Dec. 2003.

VIOLA, M. R. Simulação hidrológica na cabeceira da Bacia Hidrográfica do Rio Grande de cenários de usos do solo e mudanças climáticas A1B. Tese (Doutorado em Recursos Hídricos em Sistemas Agrícolas). Universidade Federal de Lavras. Lavras, p. 286. 2011.

VIOLA, M. R.; MELLO, C. R. de; GIONGO, M.; BESKOW, S.; SANTOS, A. F. dos. Modelagem hidrológica em uma sub-bacia hidrográfica do baixo rio Araguaia, TO. Journal of Biotechnology and Biodiversity, v. 3, n. 3, p. 38-47, 2012.

VIOLA, M. R.; MELLO, C. R. de; BESKOW, S.; NORTON, L. D. Impacts of land-use changes on the hydrology of the Grande river basin headwaters, Southeastern, Brazil. Water Resources Management, v. 28, n. 13, p. 4537-4550, 2014.

WHEATER, H. S. Modeling hydrological processes in arid and semi-arid areas: in introduction to the workshop. In: WHEATER, H. S.; SOROOSHIAN, S.; SHARMA, K. D. (Ed.). Hydrological modeling in arid and semi-arid areas. New York: Cambridge University, 2008. p. 1-20.

WHITE, K. L.; CHAUBEY, I. Sensitivity Analysis, Calibration and Validation for a Multisite and Multivariable SWAT Model. Journal of the American Water Resources Association, p. 10771089, 2005.

WORLD METEOROLOGICAL ORGANIZATION. Guide to Hydrological Practices. Volume II: Management of Water Resources and Application of Hydrological Practices. WMO No. 168. 6a ed. WMO: Geneva, Switzerland, 2009. 302 p.

XIONG, L.; O'CONNOR, K. M. Analysis of the response surface of the objective function by the optimum parameter 
curve: how good can the optimum parameter values be? Journal of Hydrology, v. 234, n. 3-4, p. 187-207, 2000.

YUAN, Y., KHARE, Y., WANG, X., PARAJULI, P B., KISEKKA, I., FINSTERLE, S. Hydrologic and water quality models: Sensitivity. Transactions of the ASABE, v. 58, n. 6, p. 1721-1744, 2015.

ZECKOSKI, R. W.; SMOLEN, M. D.; MORIASI, D. N.; FRANKENBERGER,; J. R. FEYEREISEN, G. W. Hydrologic and water quality terminology as applied to modeling. Transactions of the ASABE, v. 58, n. 6, p. 1619-1635, 2015. 